\title{
Novel 'split bladder' model to study micturition reflex in rats
}

The mechanisms governing development of diseases causing lower urinary tract disorders, such as overactive bladder and bladder pain syndrome/interstitial cystitis, remain unclear. Although several mechanisms have been proposed, currently available experimental models to investigate such mechanisms are insufficient. However, this situation could be set to change with the development of a novel rat model enabling investigation of the effects of stretch, electrical and pharmacological stimulation of the bladder in vivo and in situ.

Researchers led by Patrik Aronsson of the University of Gothenberg, Sweden, developed and characterized an in situ model for studying both afferent and efferent levels of the micturition reflex. Rats were anaesthetized and the urinary bladder dissected and separated into two halves along the midline, right down to the urethra. One of the separated bladder halves could then be used for applying stimulation and the other for measurement of detrusor muscle tension. "There has long been a need for new physiologically relevant in vivo/in situ models to study pathological changes of the reflex arc in the lower urinary tract," Aronsson commented to Nature Reviews Urology. The team compared the data generated with the same experiments carried out in a conventional whole bladder model, using stimulation with electrical impulses and agonists including ATP and methacholine, as well as mechanical stretch.

\section{4....mechanical stretch of one half of the bladder evoked contractile responses in the contralateral half... 77}

Intravenous injection of ATP and methacholine into the femoral vein evoked dose-dependent contraction of the bladder in both the conventional and the novel model. Similar results were also observed when the models were tested using electrical stimulation of the efferent part of the pelvic nerve. Cutting the pelvic nerve distally to the placement of the electrode also enabled the team to assess the effect of stimulating the afferent fibres of the nerve. Interestingly, mechanical stretch of one half of the bladder evoked contractile responses in the contralateral half, despite there being no anatomical connection between the two.

The in situ model has several advantages over conventional whole bladder techniques, enabling examination of stretchinduced contractions, as well as of the reflex arc by electrical nerve stimulation, whilst producing comparable data to whole bladder models. It also enables researchers to manipulate the part of the bladder being stretched (for example by removing the urothelium), while keeping the contractile half of the bladder intact, and could be useful for investigating both inflammatory and neurological bladder disorders.

Annette Fenner

Original article Aronsson, P. et al. A novel in situ urinary bladder model for studying afferent and efferent mechanisms in the micturition reflex in the rat. Neurourol. Urodyn. doi:10.1002/nau.22435 